\title{
Evidence to service gap: cardiac rehabilitation and secondary prevention in rural and remote Western Australia
}

\author{
Sandra Hamilton ${ }^{1,3^{*}}$, Belynda Mills', Shelley McRae ${ }^{2}$ and Sandra Thompson ${ }^{1}$
}

\begin{abstract}
Background: Cardiovascular disease (CVD), a leading cause of morbidity and mortality, has similar incidence in metropolitan and rural areas but poorer cardiovascular outcomes for residents living in rural and remote Australia. Cardiac Rehabilitation (CR) is an evidence-based intervention that helps reduce subsequent cardiovascular events and rehospitalisation. Unfortunately CR attendance rates are as low as 10-30\% with rural/remote populations underrepresented. This in-depth assessment investigated the provision of CR and secondary prevention services in Western Australia (WA) with a focus on rural and remote populations.

Methods: CR and Aboriginal Community Controlled Health Services were identified through the Directory of Western Australian Cardiac Rehabilitation and Secondary Prevention Services 2012. Structured interviews with CR coordinators included questions specific to program delivery, content, referral and attendance.

Results: Of the 38 CR services identified, $23(61 \%)$ were located in rural $(n=11,29 \%)$ and remote $(n=12,32 \%)$ regions. Interviews with coordinators from 34 CR services (10 rural, 12 remote, 12 metropolitan) found $77 \%$ of rural/remote services were hospital-based, with no service providing a comprehensive home-based or alternative method of program delivery. The majority of rural (60\%) and remote (80\%) services provided CR through chronic condition exercise programs compared with $17 \%$ of metropolitan services; only $27 \%$ of rural/remote programs provided education classes. Rural/remote coordinators were overwhelmingly physiotherapists, and only $50 \%$ of rural and $33 \%$ of remote programs had face-to-face access to multidisciplinary support. Patient referral and attendance rates differed greatly across WA and referrals to rural/remote services generally numbered less than 5 per month. Program evaluation was reported by $33 \%$ of rural/remote coordinators.

Conclusion: Geography, population density and service availability limits patient access to CR services in rural/remote WA. Current inadequacies in delivering comprehensive centre-based CR in rural/remote settings impedes management of cardiovascular risk and opportunities for event reduction. Health pathways that ensure referral and continuity of care are needed, with emerging technology-based CR support to supplement centre-based CR services requiring assessment. Implementing systematic data collection across services to establish benchmarks and enable service monitoring and evaluation is needed.
\end{abstract}

Keywords: Cardiac rehabilitation, Secondary prevention, Rural and remote, Western Australia, Access, Health services, Alternative methods

\footnotetext{
*Correspondence: sandy.hamilton@uwa.edu.au

${ }^{1}$ Western Australian Centre for Rural Health, University of Western Australia,

35 Stirling Highway, Crawley, WA 6009, Australia

${ }^{3}$ Western Australian Centre for Rural Health, PO Box 109, Geraldton, WA 6531,

Australia

Full list of author information is available at the end of the article
} 


\section{Background}

Cardiovascular disease (CVD) is a leading cause of morbidity and mortality in Australia and the leading disease category for health-care expenditure [1, 2]. In 2011, CVD accounted for $31 \%$ of all Australian deaths, with coronary heart disease (CHD) accounting for $15 \%$ of all deaths and $47 \%$ of CVD deaths, many premature and preventable [3].

Although metropolitan and rural levels of cardiovascular disease (CVD) are similar, cardiovascular outcomes for rural and remote people are poorer [4]. Rural and especially remote regions of Australia have a substantially higher proportion of Aboriginal and Torres Strait Islander (hereafter Aboriginal) people [5], a group with higher rates and earlier onset of CVD compared with non-Aboriginal people [6, 7]. Vulnerable patient populations and those with multiple comorbidities are less likely to receive, adhere to and complete cardiac rehabilitation $[8,9]$, leading to suboptimal secondary prevention and clinical benefit.

Cardiac rehabilitation (CR) is a coordinated multidimensional evidence-based strategy that aims to assist patients with CVD return to "an active and satisfying life and to prevent the recurrence of cardiac events" [10]. Secondary prevention of CVD (hereafter referred to as secondary prevention) is defined as "healthcare designed to prevent recurrence of cardiovascular events or complications of CVD in patients diagnosed with CVD" [11]. Although these definitions are similar, CR may be time limited, whereas secondary prevention proposes a cardiac rehabilitation continuum where care is provided for the rest of a person's life according to need [11].

$\mathrm{CR}$ results in improved clinical and behavioural outcomes for patients with CVD $[8,12,13]$. Clinical benefits include improved cardiovascular risk factor profile and symptoms, better adherence to pharmacotherapy, improved exercise tolerance, a reduction in readmissions for myocardial infarction or revascularisation procedures, and a $25 \%$ reduction in mortality $[8,13,14]$. Improved psychosocial wellbeing and quality of life [13], reduced levels of stress, depression and cigarette smoking have also been demonstrated $[8,14,15]$. In Western Australia (WA), more than $40 \%$ of CHD events, $50 \%$ of CHD death and $35 \%$ of nonfatal myocardial infarctions occur in people with existing CHD [16].

Unfortunately, attendance rates at traditional CR programs, both globally and in Australia, are estimated to be as low as $10-30 \%$ with rural, remote and Aboriginal populations under-represented [8, 10, 12]. Low attendance rates reflects both healthcare service-related factors (health professionals and health care service and systems) and patient-related barriers to attendance or adherence despite referral [17-21]. Barriers and contributory factors are documented in Table 1.

Barriers to CR attendance for rural, remote and Aboriginal patients are similar but of greater magnitude $[18,22]$. A cross-sectional survey of Aboriginal cardiovascular patients and Aboriginal Health Workers in remote Queensland identified large family size and family support, post-surgical pain, lack of knowledge about $\mathrm{CR}$, and low income as barriers to CR attendance [22]. A Canadian study examined CR barriers by rurality and socioeconomic status and demonstrated that rural patients attended significantly fewer CR sessions and had significantly greater barriers compared to urban residents: the most prominent barriers were distance, cost and transportation issues [18]. Brual et al. assessed the effect of drive time on CR service utilization and found that a drive time of $60 \mathrm{~min}$ or more influenced patient referral and enrolment [23].

Table 1 Barriers to cardiac rehabilitation

\begin{tabular}{|c|c|c|}
\hline & Barrier & Contributory factor \\
\hline \multirow{3}{*}{$\begin{array}{l}\text { Healthcare Professional / } \\
\text { Health Service related } \\
\text { Referral and access }\end{array}$} & Referral failure & $\begin{array}{l}\text { Clinician awareness and recommendation, inadequate referral pathways, } \\
\text { lack of a dedicated CR coordinator, vulnerable patient populations. }\end{array}$ \\
\hline & Program inflexibility & $\begin{array}{l}\text { Accessibility, inflexible program hours, failure to meet individual needs, } \\
\text { geographical location (distance, time and transport difficulties). }\end{array}$ \\
\hline & Program availability & $\begin{array}{l}\text { Absence of local program, limited program places, fragmented funding, } \\
\text { geographical location. }\end{array}$ \\
\hline \multirow[t]{6}{*}{ Patient related } & Access to services: & Transport, work, social/family, distance, financial, geographical \\
\hline & Patient perception & $\begin{array}{l}\text { Need, value, knowledge, futility, views of health system, financial } \\
\text { considerations }\end{array}$ \\
\hline & $\begin{array}{l}\text { Lack of motivation, or commitment } \\
\text { or adherence }\end{array}$ & Lack of energy, functional impairment, vulnerable patients, smoking \\
\hline & Vulnerable patient populations: & $\begin{array}{l}\text { Aboriginal, other ethnic minority populations, women, rural patients, } \\
\text { older patients, comorbidities, socio-economically disadvantaged }\end{array}$ \\
\hline & Lack of support & Health Professionals, family, social \\
\hline & Type of cardiac event & Patients following $\mathrm{AMI}$ and $\mathrm{PCI}$ less likely to attend than $\mathrm{CABG}$ \\
\hline
\end{tabular}


Given the service and patient related barriers to traditional CR, a diverse array of alternative models of CR have been developed, including multifactorial patientcentred telehealth and community- or home-based CR, and can be preferred by many patients [15, 24]. These programs offer flexible and individualised management of CVD through in-person visits, community programs and home manuals with mobile support via phone or other electronic means [25]. These have produced similar reductions in CVD risk factors compared with traditional outpatient CR [15]. Following a 2004 review of the evidence for non-conventional CR models of care, Dollard et al., concluded that home-based CR had the greatest potential for improving accessibility to CR for rural and remote patients [26]. However, there remains a paucity of evidence for the adaptability and effectiveness of these programs in rural, remote and Aboriginal patients [15].

This in-depth assessment mapped CR and secondary prevention services in WA to inform future work investigating the processes and systems of referral and the implementation of alternative methods of CR delivery in rural and remote settings.

\section{Methods}

\section{Study design}

A mixed methods in-depth study of CR services in WA was undertaken from March to July 2015 and included the following agencies: North and South Metropolitan Health Services, West Australian Country Health Service (hospital and community care programs); private physiotherapy providers; and primary care including Aboriginal Community Controlled Health Organisations (ACCHOs).

A structured interview guide was developed by the first author in consultation with the co-authors and piloted with a local CR coordinator before use. Quantitative and open-ended interview questions (Table 2) were based on a previous survey which examined CR and secondary prevention for Aboriginal people post discharge for Acute Coronary Syndrome [27]. However, this was broadened and adapted to incorporate main elements for inpatient and outpatient CR as documented in the National Heart Foundation of Australia and the Australian Cardiac Rehabilitation Association Recommended Framework for Cardiac Rehabilitation 04 [10]. Quantitative data was based on documentation of CR coordinator reports at interview, with a summary of service data forwarded by email wherever possible.

Cardiac rehabilitation services and program coordinators were identified through the Heart Foundation (HF) Directory of Western Australian Cardiac Rehabilitation and Secondary Prevention Services 2012 [28]. This directory provides a comprehensive list of state-wide CR
Table 2 Components of cardiac rehabilitation included in the interview guide

\begin{tabular}{|c|c|}
\hline Component & Content \\
\hline $\begin{array}{l}\text { Core component of cardiac } \\
\text { rehabilitation }\end{array}$ & $\begin{array}{l}\text { Inpatient CR: Type of program; type of } \\
\text { facility; main elements of inpatient CR } \\
\text { (basic information and reassurance, } \\
\text { supportive counselling, mobilization and } \\
\text { resumption of activities, discharge } \\
\text { planning, and referral to outpatient CR } \\
\text { and ongoing care). } \\
\text { Outpatient CR: Pre-cardiovascular surgery } \\
\text { review; assessment, review and follow-up; } \\
\text { low or moderate intensity physical activity; } \\
\text { education, discussion and counselling; } \\
\text { ongoing assessment and management, } \\
\text { and monitoring and evaluation. }\end{array}$ \\
\hline Program information & $\begin{array}{l}\text { Type of health service; type of program } \\
\text { delivery; patient eligibility; program } \\
\text { duration and frequency; attendance fee; } \\
\text { patients from diverse populations; and } \\
\text { patient monitoring and referral for further } \\
\text { medical review. }\end{array}$ \\
\hline Referral and attendance & $\begin{array}{l}\text { Patient referral pathway; patient } \\
\text { attendance and completion numbers; } \\
\text { patient outcomes; and exit strategies for } \\
\text { patients on completion. }\end{array}$ \\
\hline $\begin{array}{l}\text { Program coordination and } \\
\text { multidisciplinary team }\end{array}$ & $\begin{array}{l}\text { Dedicated program coordinator; } \\
\text { multidisciplinary team availability; use of } \\
\text { cardiac rehabilitation guidelines; } \\
\text { sustainability of program and program } \\
\text { and service contact details. }\end{array}$ \\
\hline
\end{tabular}

Key: $C R$ Cardiac Rehabilitation

services in WA. Identified services were contacted and coordinators invited to participate, given verbal and written information about the study. Interviews were scheduled at the coordinator's convenience following informed consent. Interview responses were documented and digitally recorded with the coordinator's consent.

The Western Australian Country Health Service and University of Western Australia Human Ethics Committees approved the study.

\section{Data analysis}

Quantitative data was analysed using IBM SPSS Statistics 22 with descriptive statistics to report both continuous and categorical data. Qualitative data was analysed manually to identify program content, concepts and themes in relation to the research aims. The Australian Government Department of Health's Rural, Remote and Metropolitan Areas (RRMA) classification was utilised to classify service location. The RRMA classification is based on Statistical Local Areas (SLA) and based primarily on population numbers and an index of remoteness (Table 3) [29].

\section{Results}

In total 70 services were identified from the CR Directory, including 18 ACCHOs. Of these, 38 services 
Table 3 Structure of the Rural, Remote and Metropolitan Areas (RRMA) classification [29]

\begin{tabular}{|c|c|c|}
\hline Zone & & Class \\
\hline \multirow[t]{2}{*}{ Metropolitan zone } & M1 & Capital cities \\
\hline & M2 & $\begin{array}{l}\text { Other metropolitan centres (urban } \\
\text { centre population } \geq 100,000 \text { ) }\end{array}$ \\
\hline \multirow[t]{3}{*}{ Rural zone } & R1 & $\begin{array}{l}\text { Large rural centres (urban centre } \\
\text { population } 25,000-99,999 \text { ) }\end{array}$ \\
\hline & R2 & $\begin{array}{l}\text { Small rural centres (urban centre } \\
\text { populations 10,000-24,999) }\end{array}$ \\
\hline & R3 & $\begin{array}{l}\text { Other rural centres (urban centre } \\
\text { populations }<10,000 \text { ) }\end{array}$ \\
\hline \multirow[t]{2}{*}{ Remote zone } & Rem1 & $\begin{array}{l}\text { Remote centres (urban centre } \\
\text { population } \geq 5000 \text { ) }\end{array}$ \\
\hline & Rem2 & $\begin{array}{l}\text { Other remote centres (urban centre } \\
\text { population < 5000) }\end{array}$ \\
\hline
\end{tabular}

provided a $\mathrm{CR} /$ secondary prevention program, 12 had discontinued $\mathrm{CR}$ programs and $14 \mathrm{ACCHOs}$ did not offer CR or heart health programs. Difficulty in establishing contact meant it is unknown for 6 services what CR if any they offer (Table 4). Thirty four Cardiac Rehabilitation Coordinators were interviewed from March to July 2015: four who were contacted declined interview due to work load constraints.

\section{Geographical location of services}

From the 34 services where a representative was interviewed, 22 (65\%) were located in larger rural and remote towns or regional cities. The remaining 12 (35\%) services were located in metropolitan Perth (Table 5). Of the 22 rural and remote programs, 11 (50\%) were located in the health regions of southern WA: the Wheatbelt, South West, and Great Southern. The remaining 50\% were based in the Kimberley $(n=3)$, Pilbara $(n=3)$, Midwest $(n=3)$, and Goldfields $(n=2)$, an area of some $2,312,237 \mathrm{~km}^{2}$ or $87 \%$ of the total WA land mass.

\section{Inpatient cardiac rehabilitation service provision}

Eight of 34 coordinators reported responsibilities for inpatient CR (Table 5); all programs offered individualised patient-centred inpatient CR. Metropolitan programs were more likely to offer all the main elements of inpatient CR (Table 2) than rural and remote programs.

\section{Outpatient cardiac rehabilitation service provision}

All 34 services provided outpatient CR, 68\% hospitalbased, 29\% community-based and one private provider was both hospital- and community-based (Table 5). No coordinator offered comprehensive home-based or an alternative method of program delivery. A minority (18\%) of coordinators reported offering a home program, home exercise, or a graduated walking program; in one instance, this was for patients who did not want to attend group sessions. Other methods of support included telephone support, home visits if required (metropolitan private service and rural and remote programs) with two $\mathrm{CR}$ coordinators in remote settings reporting visits to Aboriginal communities.

Program length varied from 6 to 24 weeks, with some services offering an ongoing program. Rural and remote programs $(45 \%)$ were more likely to have an ongoing access policy compared with $25 \%$ of metropolitan programs. Most classes (exercise or education) were run on set days and at set times. Flexibility if available occurred through an option of days or extended hours on a set days. One program reported an after-hours education class which improved attendance. Where comprehensive programs were delivered, these included exercise (low or moderate intensity physical activity),

Table 4 Identified programs using the Heart Foundation Directory 2012

\begin{tabular}{|c|c|c|c|c|}
\hline & Overall $n$ & Rural $n(\%)$ & Remote $n(\%)$ & Metropolitan $n(\%)$ \\
\hline \multicolumn{5}{|c|}{ All services listed in Heart Foundation Directory } \\
\hline Total & 70 & $16(23)$ & $30(43)$ & $24(34)$ \\
\hline No Program & 14 & $1(7)$ & $13(93)$ & 0 \\
\hline Program Discontinued & 12 & $3(25)$ & $2(17)$ & $7(58)$ \\
\hline Unknown & 6 & $1(17)$ & $3(50)$ & $2(33)$ \\
\hline Program offered & 38 & $11(29)$ & $12(32)$ & $15(39)$ \\
\hline Interviewed & 34 & $10(30)$ & $12(35)$ & $12(35)$ \\
\hline \multicolumn{5}{|l|}{ Aboriginal Medical Services } \\
\hline Total & 18 & $2(11)$ & $15(83)$ & $1(6)$ \\
\hline Program offered & 2 & $1(50)$ & $0(0)$ & 1(50) \\
\hline No Program & 14 & $1(7)$ & $13(93)$ & $0(0)$ \\
\hline Unknown & 2 & $0(0)$ & $2(100)$ & $0(0)$ \\
\hline Interviewed & 2 & $1(1)$ & $0(0)$ & $1(1)$ \\
\hline
\end{tabular}


Table 5 Cardiac rehabilitation service provision in Western Australia

\begin{tabular}{|c|c|c|c|}
\hline & $\begin{array}{l}\text { Rural } \\
n(\%)\end{array}$ & $\begin{array}{l}\text { Remote } \\
n(\%)\end{array}$ & $\begin{array}{l}\text { Metropolitan } \\
n(\%)\end{array}$ \\
\hline CR service location & $10(30)$ & $12(35)$ & $12(35)$ \\
\hline Public inpatient CR service & $3(30)$ & $1(8)$ & $3(25)$ \\
\hline Private inpatient $C R$ service & 0 & 0 & $1(8)$ \\
\hline $\begin{array}{l}\text { Public hospital-based outpatient } \\
\text { CR service }\end{array}$ & $8(80)$ & $9(75)$ & $6(50)$ \\
\hline $\begin{array}{l}\text { Public community-based } \\
\text { outpatient CR service }\end{array}$ & $2(20)$ & $3(25)$ & $5(42)$ \\
\hline $\begin{array}{l}\text { Private hospital and community- } \\
\text { based CR service }\end{array}$ & 0 & 0 & $1(8)$ \\
\hline Supervised exercise classes & $10(100)$ & $11(92)$ & $12(100)$ \\
\hline Comprehensive education classes & $3(30)$ & $2(17)$ & $7(58)$ \\
\hline $\begin{array}{l}\text { Generic chronic condition } \\
\text { rehabilitation classes }\end{array}$ & $6(60)$ & $10(80)$ & $2(17)$ \\
\hline Multidisciplinary $C R$ team care & $5(50)$ & $4(33)$ & $8(67)$ \\
\hline CR program evaluation & $4(40)$ & $4(33)$ & $9(75)$ \\
\hline CR guideline use & $6(60)$ & $8(67)$ & $12(100)$ \\
\hline
\end{tabular}

education, psychosocial support, self-management support, and management of symptoms and medications. Most programs offered supervised exercise classes at a low and/or moderate intensity; one remote nursing post offered telephone liaison, support and education. Many programs were unable to offer formal education classes and in these instances informal education was given at all exercise classes. Programs in rural and remote settings were more likely to be generic chronic condition rehabilitation compared with metropolitan programs (Table 5).

Table 6 shows the program delivery characteristics of program coordinators who were generally physiotherapists or registered nurses and more likely to be female (75\%) regardless of rurality. All rural and $83 \%$ of remote program coordinators were physiotherapists; CR coordinators in rural and remote programs had only a part time CR role generally as only one component of the person's overall role. The length of time of coordinators had worked in their role varied, with a wide range in all program settings. Multidisciplinary CR team care was more likely in metropolitan programs (Table 5), with rural and remote programs having the most challenges accessing multidisciplinary input which often had to occur by telephone.

\section{Referral and attendance}

Western Australia (and Australia) has no standard system of CR data collection so our quantitative analysis is limited, based on coordinator reports rather than analysis of consistent, routinely collected service data. All program coordinators reported receiving patient referrals from metropolitan public hospitals. Table 7 shows other referral sources. Cardiac rehabilitation coordinators, nursing and allied health staff and cardiologists were most likely to make the referral and the most common method of referral was a referral letter or a copy of the discharge letter. Other methods of referral included telephone calls, facsimile, eReferral and email. The timeliness to receipt of referral following discharge varied, with $46 \%$ of referrals received at the time of discharge with others following in the ensuing weeks [1-2 weeks (15\%), 3-4 weeks (18\%), 1-3 months (12\%) and other (9\%)].

Referral numbers varied widely and were greater for metropolitan services. The majority of rural and remote services received less than 5 cardiac referrals per month, with some remote services receiving less than 5 per year. In comparison, referral rates at metropolitan services varied from 2 to 105 per month, with higher referral rates at tertiary hospitals, a private cardiovascular prevention and rehabilitation service (73-105 per month) and a larger metropolitan community service (47 per month). Heart Failure services and smaller community services received 2 to 27 referrals per month.

Table 6 Program delivery characteristics of cardiac rehabilitation coordinators

\begin{tabular}{|c|c|c|c|c|}
\hline & Overall $n(\%)$ & Rural $n(\%)$ & Remote $n(\%)$ & Metropolitan $n(\%)$ \\
\hline \multicolumn{5}{|c|}{ Health discipline of CR Coordinator } \\
\hline Registered nurse & $8(24)$ & & $2(17)$ & $6(50)$ \\
\hline Physiotherapist & $23(67)$ & $10(100)$ & $10(83)$ & $3(25)$ \\
\hline Exercise Physiologist & $2(6)$ & & & $2(17)$ \\
\hline Health Promotion officer & $1(3)$ & & & $1(8)$ \\
\hline \multicolumn{5}{|c|}{ Full time equivalent (FTE) in CR role } \\
\hline$\leq 0.8$ FTE (range $0.2-0.8$ ) & $27(79)$ & $10(100)$ & $12(100)$ & $5(42)$ \\
\hline 1.0 FTE & $7(21)$ & $0(0)$ & $0(0)$ & $7(58)$ \\
\hline \multicolumn{5}{|l|}{ Months in position } \\
\hline mean $\pm S D$ & $52.8 \pm 67.7$ & $65.1 \pm 97.2$ & $48.5 \pm 58.7$ & $47.8 \pm 53.4$ \\
\hline Range (min-max) & $1-276$ & $3-276$ & $1-192$ & $3-192$ \\
\hline
\end{tabular}


Table 7 Referral source

\begin{tabular}{lc}
\hline & $\begin{array}{c}\text { \% of CR Coordinators } \\
\text { reporting referral source }\end{array}$ \\
\hline Metropolitan public hospitals & 100 \\
Regional public hospitals & 50 \\
Metropolitan private hospitals & 60 \\
Regional private hospitals & 35 \\
General practice & 60 \\
Aboriginal Medical Services & 50 \\
Private cardiology rooms & 3 \\
Self-referrals & 40 \\
Aboriginal community clinics. & 17 \\
\hline
\end{tabular}

The percentage of those patients who were referred to $\mathrm{CR}$ and then attended was reported by $70 \%$ of the CR Coordinators. Attendance rates were similar across regions (Table 8 ). Two thirds of patients who attended CR were male (Table 8). Limited information was available for patient completion rates with only $35 \%$ of coordinators $(n=12)$ reporting data. Of these, 9 coordinators reported completion rates (defined as completing $>75 \%$ of program sessions) of $75-100 \%$.

\section{Data collection, documentation and evaluation}

All coordinators reported conducting biomedical and exercise assessments, monitoring progress and documenting findings and patient progress. However, no standard system of data collection was identified. The most common (65\%) method of documentation was reporting the patient's progress in their medical record. Other methods of documentation included: assessment sheets $(n=3)$; nursing notes $(n=1)$; traditional physiotherapy notes $(n=2)$; SF36 form $(n=2)$; an attendance record $(n=4)$; own data base $(n=3)$; Cardiobase $(n=2)$; completion letters $(n=2)$ and a check list at group sessions $(n=1)$. Two programs (CR and Heart Failure) at one metropolitan tertiary hospital used multiple methods of the data documentation described above.

A third of coordinators reported undertaking patient evaluation which was described as administering a patient evaluation form (25\%) or a patient satisfaction survey (8\%). Program evaluation was reported by $50 \%$ of coordinators and included quarterly or yearly reports to a funding body or management, evaluation of improvement in patient risk factors and outcome measures and evaluation of education classes. Evaluation frequency was variable and was reported to occur quarterly, 6 monthly, yearly to two yearly depending on service requirements.

\section{Cardiac rehabilitation guideline use}

Overall, 77\% of coordinators reported that their program was based on CR guidelines (Table 5). The National Heart Foundation of Australia and Australian Cardiac Rehabilitation Association Recommended Framework for Cardiac Rehabilitation '04 [10] was the most frequently utilised guideline. Table 9 documents other guideline utilization. A minority (9\%) of coordinators were unsure of the guidelines utilised and $6 \%$ stated that their program was "not really" guideline based.

\section{Discussion}

The recommended core components of cardiovascular disease secondary prevention and cardiac rehabilitation in Australia suggest that it is essential for all CR services to provide the most comprehensive service possible within resource availability [30]. This in-depth assessment of CR services in WA found a service gap in delivery of comprehensive $\mathrm{CR}$ and secondary prevention for people residing in rural and remote settings, reflecting limitations related to geographical location, service design and comprehensiveness, health professional availability, referral pathways and numbers. Data collection is inconsistent and given inadequate attention, limiting program evaluation and the potential for improvements in quality.

The delivery of comprehensive CR programs in rural and remote WA is challenged by geographical location, low population density and limited service availability. Sixteen percent of the Australian population live in geographical regions with poor or no access to CR and secondary prevention services [31]. Distance, transport difficulties, cost and family responsibilities are significantly greater barriers to CR access for rural compared with metropolitan patients [18]. Most CR services in rural/remote WA are located in regional centres, creating access challenges related to transport, time and cost for residents outside these centres. We have demonstrated lower CR referral rates in rural and remote WA compared with metropolitan rates, in part reflecting a lower population density. Referral failure is a known

Table 8 Percent attendance of referred patients

\begin{tabular}{|c|c|c|c|c|c|c|c|c|c|}
\hline & \multicolumn{3}{|l|}{ Rural } & \multicolumn{3}{|l|}{ Remote } & \multicolumn{3}{|c|}{ Metropolitan } \\
\hline & Mean \% & Range \% & $\overline{S D}$ & Mean \% & Range \% & $\overline{S D}$ & Mean \% & Range \% & $\overline{S D}$ \\
\hline \multirow[t]{2}{*}{ Referred patients who attended } & 75 & $33-100$ & 29.2 & 75 & $50-100$ & 22.9 & 72 & $30-100$ & 29.7 \\
\hline & & $\%$ & & & $\%$ & & & $\%$ & \\
\hline Male & & 60 & & & 66 & & & 60 & \\
\hline
\end{tabular}


Table 9 Cardiac rehabilitation guideline use

\begin{tabular}{|c|c|}
\hline & $\begin{array}{l}\% \text { of CR Coordinators } \\
\text { reporting use }\end{array}$ \\
\hline $\begin{array}{l}\text { National Heart Foundation of Australia and } \\
\text { Australian Cardiac Rehabilitation Association } \\
\text { Recommended Framework for Cardiac } \\
\text { Rehabilitation '04 [10] }\end{array}$ & 60 \\
\hline $\begin{array}{l}\text { The Australian Cardiovascular Health and } \\
\text { Rehabilitation Association Core Components of } \\
\text { Cardiovascular Disease Secondary Prevention and } \\
\text { Cardiac Rehabilitation } 2014 \text { [30] }\end{array}$ & 15 \\
\hline $\begin{array}{l}\text { Department of Health, Western Australia, } \\
\text { Cardiovascular rehabilitation and secondary } \\
\text { prevention pathway principles for Western } \\
\text { Australia, } 2014 \text { [32] }\end{array}$ & 15 \\
\hline $\begin{array}{l}\text { National Health and Medical Research Council, } \\
\text { Strengthening Cardiac Rehabilitation and } \\
\text { Secondary Prevention for Aboriginal and Torres } \\
\text { Strait Islander Peoples: A Guide for Health } \\
\text { Professionals [38]. }\end{array}$ & 0 \\
\hline $\begin{array}{l}\text { Utilization of more than one guideline, including } \\
\text { WA Models of Care and interstate or } \\
\text { international programs/guidelines }\end{array}$ & 20 \\
\hline
\end{tabular}

barrier to CR enrolment and attendance [17-20], however, Brual et al. report that a drive time of greater than 60 min effects CR referral and enrolment but not attendance once enrolled [23]. This highlights the importance of effective referral systems, pathways and offering alternative means of CR service delivery for cardiac patients at greater geographic disadvantage.

Traditional service delivery for $\mathrm{CR}$ is centre-based and ideally it incorporates a comprehensive program of assessment, education and self-management strategies to promote behaviour change, exercise, psychosocial support, medical follow-up and service evaluation and quality improvement $[25,30,32]$. However, alternative models of CR utilising patient-centred telehealth and technology (internet and mobile health via smartphone apps) and supporting home-based CR have been developed and are preferred by many patients in all geographical settings [15, 24, 33, 34]. Despite these advances, in rural/remote WA the majority of CR services were centre-based exercise classes with informal education or chronic condition rehabilitation classes that accommodates the cardiac patient. A fifth of coordinators reported the capacity to provide support for home exercise but there was no comprehensive home-based or alternative method of program delivery provided. Efforts to utilise more modern approaches based on advances in technology to delivery of $\mathrm{CR}$ in rural and remote WA are needed. Mobile health technology for the delivery of a comprehensive $\mathrm{CR}$ program demonstrated improved uptake and completion rates and improved health outcomes equal to centre-based CR in a Brisbane study $[35,36]$. Implementation of such technology has great potential for overcoming the challenges to service delivery associated with geographical location and isolation. However, its benefits are still to be tested in rural and remote settings and its utility to support Indigenous people remains to be assessed.

Given the complexity of a cardiac patient's recovery, $\mathrm{CR}$ is ideally delivered by a multidisciplinary team with appropriate qualifications and expertise [30]. Less than half of rural/remote services reported the capacity to provide face-to-face multidisciplinary team care, so that access to multidisciplinary expertise was often by telephone. In rural and remote programs, coordination of $\mathrm{CR}$ was only one component of multiple roles for those who were designated coordinators $(90 \%$ of whom were physiotherapists) and this, together with the centrebased nature of the program and limited access to a multidisciplinary team, limited program comprehensiveness and flexibility.

Thirty percent of services listed in the Heart Foundation Directory either did not provide CR or the program had been discontinued, suggesting considerable service turnover. All 14 services that were unable to provide a $\mathrm{CR}$ program were rural and remote ACCHOs. Although the reasons for this is unclear, it possibly relates to multiple factors such as geographical context, population density, provision of CR by the Country Health Service in some locations, ACCHO funding cycles and staff recruitment and retention. Program discontinuation in rural and remote regions in two instances (and possibly a further two) was related to the "rationalisation" of the service to an outreach physiotherapy service on a once per month basis. One rural private physiotherapy clinic discontinued CR. Discontinuation of metropolitan services related to withdrawal of telephone $C R$ coaching and restructuring of $\mathrm{CR}$ services with the opening of a new tertiary hospital.

Ongoing attention to improvement of $\mathrm{CR}$ services and the provision of flexible, accessible and guidelinebased alternative methods of CR delivery in rural areas is needed in order to improve access to $C R$ for rural and remote patients in WA. Accessibility requires that all dimensions of access as described by Levesque et al., are considered and encompass approachability, acceptability, availability and accommodation, affordability and appropriateness (including cultural appropriateness [37]. The relatively high level of completion of CR described by coordinators indicates that an essential step in $\mathrm{CR}$ delivery is referral and initial attendance. Endorsement of guideline implementation, and a structured integrated health pathway to ensure coordinated referral and management of CR and secondary prevention are essential. Raising awareness and quality professional development for all health professionals involved in supporting CVD patient journeys could improve knowledge and use of CR guidelines. 


\section{Strengths and limitations}

The strength of our study relates to the in-depth assessment undertaken, the rich level of data obtained from the CR coordinators and high participation of services in the study. While the Heart Foundation (HF) Directory of Western Australian Cardiac Rehabilitation and Secondary Prevention Services 2012 is a comprehensive directory of CR services and a small number of new services were known and contacted, it remains possible that a small number of services were not identified. A further limitation is the inability to estimate the number of patients who are not referred to CR, as access to concurrent discharge data for the number of eligible patients was not included in the study.

CR services in WA currently lack a standard method of data collection, limiting availability and analysis of quantitative and qualitative client data. A variety of data collection and evaluation methods were used by services across WA, so data available differed across services, and was particularly deficient for those in rural and remote areas. Inconsistency of quantitative data collection across services precluded comparisons between regions and comparative statistical analysis. The inconsistency of data collection for referral, attendance and completion rates also prevented a per capita comparison between rural, remote and metropolitan services. Population density is lower in rural and remote regions leading to lower referral numbers, however, most rural and remote CR services were situated in larger towns or regional cities. This means those living outside of regional centres have longer travel times and costs in order to attend, with time and distance being a strong influence on referral and enrolment rates [23]. Australian guidelines recommend the collection of information to identify problem areas and measure change [30, 38]. Implementing a minimum dataset to record and monitor $\mathrm{CR}$ and secondary prevention in WA $[11,32]$ would enable evaluation of the quality and outcome of participation in each of the five core components of CR [30]. A nationally agreed minimum data set, if implemented at a national level, could enable service performance indicators for all phases of CR; tracking patient referral, attendance, adherence, completion rates and outcomes. Establishing consistent relevant benchmarks will help ensure greater accountability for service function in rural/remote settings.

\section{Conclusions}

Geography, population density and service availability limits patient access to CR services in rural/remote WA. Current inadequacies in comprehensive centre-based CR in rural/remote settings impedes management of cardiovascular risk and opportunities for event reduction. Health pathways that ensure referral and continuity of care are recommended, with implementation and evaluation of emerging technology-based CR support to add value to centre-based $C R$ services. Implementing systematic data collection across services to establish benchmarks and enable service monitoring and evaluation is an essential component of progress. Future work is required by linking discharge data to assess and improve the proportion of eligible patients referred to $\mathrm{CR}$ and so that attention is given to addressing disparities between metropolitan, rural and remote settings.

\section{Abbreviations}

ACCHO: Aboriginal community controlled health organisation; CHD: Coronary heart disease; CR: Cardiac rehabilitation; CVD: Cardiovascular disease; WA: Western Australia

\section{Acknowledgements \\ We gratefully acknowledge the health services and all the Cardiac Rehabilitation Coordinators who participated. The National Heart Foundation WA develops and maintains the Directory of Western Australian Cardiac Rehabilitation and Secondary Prevention Services.}

Funding

Dr. Hamilton is a Poche Research Fellow and acknowledges support from the UWA Poche Centre of Indigenous Health. The WA Centre for Rural Health receives core support from the Australian Department of Health.

\section{Availability of data and materials}

The dataset used and analysed for this study is held by the corresponding author. The dataset is available from the corresponding author on reasonable request and following ethical approval to share.

\section{Authors' contributions}

SH contributed to the study conception and design, secured ethical and governance approvals, stakeholder engagement, data collection, analysis, interpretation and writing. BM contributed to study design, ethical approval, stakeholder engagement, data collection and writing. SM contributed to stakeholder engagement and writing. SCT contributed to study conception and design, interpretation and writing. All authors approved the final manuscript.

\section{Ethics approval and consent to participate}

The Western Australian Country Health Service and University of Western Australia Human Ethics Committees approved the study. Coordinators signed and return the consent form and were again verbally consented at the beginning of the interview.

\section{Consent for publication}

Participants were informed in the Participant Information Form of publication and signed consent to the utilization of data as outlined in the Participant Information Form was obtained.

\section{Competing interests}

The authors declare that they have no competing interests.

\section{Publisher's Note}

Springer Nature remains neutral with regard to jurisdictional claims in published maps and institutional affiliations.

\section{Author details}

'Western Australian Centre for Rural Health, University of Western Australia, 35 Stirling Highway, Crawley, WA 6009, Australia. ${ }^{2}$ National Heart Foundation of Australia, 334 Rokeby Road, Subiaco, WA 6009, Australia. ${ }^{3}$ Western

Australian Centre for Rural Health, PO Box 109, Geraldton, WA 6531, Australia. 
Received: 9 February 2017 Accepted: 22 January 2018 Published online: 30 January 2018

\section{References}

1. Australian Bureau of Statistics: Causes of Death, Australia, 2015 (3303.0) September 2016. Canberra. 2016.

2. Australian Institute of Health and Welfare. Health-care expenditure on cardiovascular diseases 2008-09. Cat. No. CVD 65. Canberra: AlHW; 2014.

3. Australian Institute of Health and Welfare. Cardiovascular disease, diabetes and chronic kidney disease - Australian facts: mortality. Cardiovascular, diabetes and chronic kidney disease series no. 1. Cat. No.CDK 1. Canberra; 2014.

4. Australian Social Trends March 2011: Health outside major cities.

5. 2011 Census Data: Community Profiles.

6. Australian institute of Health and Welfare. Cardiovascular disease, diabetes and chronic kidney disease - Australian facts: Morbidity-Hospital care, Cardiovascular, diabetes and chronic kidney disease series no. 3. Cat. no. CDK 3. Canberra: AlHW; 2014

7. Australian Institute of Health and Welfare. Cardiovascular disease, diabetes and chronic kidney disease - Australian facts: Aboriginal and Torres Strait Islander people, Cardiovascular, diabetes and chronic disease no.5. Cat. no. CDK 5. Canberra: AlHW; 2015

8. Briffa T, Kinsman L, Maiorana A, Zecchin R, Redfern J, Davidson P, Paull G, Nagle A, Denniss A. An integrated and coordinated approach to preventing recurrent coronary heart disease events in Australia. Policy statement from the Australian cardiovascular health and rehabilitation association. Med J Aust. 2009;190(12):683-6.

9. Redfern J, Ellis E, Briffa T, Freedman S. High risk-factor level and low riskfactor knowledge in patients not accessing cardiac rehabilitation after acute coronary syndrome. Med J Aust. 2007;186(1):21-5.

10. National Heart Foundation of Australia \& Australian Cardiac Rehabilitation Association. Recommended framework for cardiac rehabilitation 04 Melbourne: National Heart Foundation of Australia; 2004. https://www. heartfoundation.org.au.

11. National Heart Foundation of Australia: Secondary prevention of cardiovascular disease; 2010

12. Leon A, Franklin B, Costa F, Balady G, Berra K, Stewart K, Thompson P, Williams M, Lauer M. Cardiac rehabilitation and secondary prevention of coronary heart disease. Circulation. 2005;111(3):369-76.

13. Anderson L, Oldridge N, Thompson DR, Zwisler A-D, Rees K, Martin N, Taylor RS. Exercise-Based Cardiac Rehabilitation for Coronary Heart Disease: Cochrane Systematic Review and Meta-Analysis. J Am Coll Cardiol. 2016; 67(1):1-12.

14. Wenger NK. Current status of cardiac rehabilitation. J Am Coll Cardiol. 2008;51(17):1619-31.

15. Clark RA, Conway A, Poulsen V, Keech W, Tirimacco R, Tideman P. Alternative models of cardiac rehabilitation: a systematic review. Eur J Prev Cardiol. 2015;22(1):35-74.

16. Briffa TG, Hobbs MS, Tonkin A, Sanfilippo FM, Hickling S, Ridout SC, Knuiman M. Population trends of recurrent coronary heart disease event rates remain high. Circ Cardiovasc Qual Outcomes. 2011:4(1):107-13.

17. Bunker S, McBurney $H$, Cox $H$, Jelinek M. Identifying participation rates at outpatient cardiac rehabilitation programs in Victoria, Australia. J Cardpulm Rehabil. 1999;19(6):334-8.

18. Shanmugasegaram S, Oh P, Reid R, McCumber T, Grace S. Cardiac rehabilitation barriers by rurality and socioeconomic status: a cross-sectional study. Int J Equity Health. 2013;12(1):72.

19. Grace SL, Gravely-Witte S, Brual J, Monette G, Suskin N, Higginson L, Alter DA, Stewart DE. Contribution of patient and physician factors to cardiac rehabilitation enrollment: a prospective multilevel study. Eur J Cardiovasc Prev Rehabil. 2008;15(5):548-56.

20. Caldwell PH, Arthur HM. The influence of a "culture of referral" on access to care in rural settings after myocardial infarction. Health \& Place. 2009:15(1):180-5.

21. Evenson KR, Fleury J. Barriers to outpatient cardiac rehabilitation participation and adherence. J Cardpulm Rehabil. 2000:20(4):241-6.

22. Shepherd F, Battye K, Chalmers E. Improving access to cardiac rehabilitation for remote indigenous clients. Aust N Z J Public Health. 2003:27(6):632-6.

23. Brual J, Gravely-Witte S, Suskin N, Stewart D, Macpherson A, Grace S. Drive time to cardiac rehabilitation: at what point does it affect utilization? Int J Health Geogr. 2010;9:27.
24. Higgins RO, Murphy BM, Grande MRL, Parkinson A, Worcester MUC, Goble AJ. Expressed preferences for health education of patients after percutaneous coronary intervention. Euro J Cardiov Prev R. 2005;12(6):572-9.

25. Redfern J, Maiorana A, Neubeck L, Clark AM, Briffa T. Achieving coordinated secondary prevention of coronary heart disease for all in need (SPAN). Int J Cardiol. 2011;146(1):1-3

26. Dollard J, Smith J, Thompson DR, Stewart S. Broadening the reach of cardiac rehabilitation to rural and remote Australia. Eur J Cardiovasc Nurs. 2004;3(1):27-42

27. Thompson S, Digiacomo M, Smith J, Taylor K, Dimer L, Ali M, Wood M, Leahy T, Davidson P. Are the processes recommended by the NHMRC for improving cardiac rehabilitation (CR) for aboriginal and Torres Strait islander people being implemented?: an assessment of CR services across Western Australia. Aust New Zealand Health Policy. 2009;6:29.

28. Heart Foundation: Directory of western Australian cardiac rehabilitation and secondary prevention services 2012.

29. DPIE (Department of Primary Industries and Energy) \& DHSH (Department of Human Services and Health). Rural, Remote and Metropolitan Areas classification. 1991 Census edition. Canberra: Australian Government Publishing Service; 1994.

30. Woodruffe S, Neubeck L, Clark RA, Gray K, Ferry C, Finan J, Sanderson S, Briffa TG. Australian cardiovascular health and rehabilitation association (ACRA) Core components of cardiovascular disease secondary prevention and cardiac rehabilitation 2014. Heart Lung Circ. 2015;24(5):430-41.

31. Clark RA, Coffee N, Turner D, Eckert KA, van Gaans D, Wilkinson D, Stewart S, Tonkin AM. Access to cardiac rehabilitation does not equate to attendance. Eur J Cardiovas Nur. 2014;13(3):235-42.

32. Department of Health WA. Cardiovascular rehabilitation and secondary prevention pathway principles for Western Australia. Perth: Health Strategy and Networks, Department of Health; 2014.

33. Widmer RJ, Collins NM, Collins CS, West CP, Lerman LO, Lerman A. Digital health interventions for the prevention of cardiovascular disease: a systematic review and meta-analysis. Mayo Clin Proc. 2015;90(4):469-80.

34. Park LG, Beatty A, Stafford Z, Whooley MA. Mobile phone interventions for the secondary prevention of cardiovascular disease. Prog Cardiovasc Dis. 2016;58(6):639-50

35. Varnfield M, Karunanithi M, Lee CK, Honeyman E, Arnold D, Ding H, Smith C, Walters DL. Smartphone-based home care model improved use of cardiac rehabilitation in postmyocardial infarction patients: results from a randomised controlled trial. Heart. 2014;100(22):1770-9.

36. Bradford D, Hansen D, Karunanithi M. Making an APPropriate program for indigenous cardiac disease: customization of an existing cardiac rehabilitation program. MEDINFO 2015: eHealth-enabled Health. 2015;216:343-7.

37. Levesque JF, Harris MF, Russell G. Patient-centred access to health care: conceptualising access at the interface of health systems and populations. Int J Equity Health. 2013;12:18.

38. National Health and Medical Research Council. Strengthening cardiac rehabilitation and secondary prevention for aboriginal and Torres Strait islander peoples: a guide for health professionals. Australia: NHMRC; 2005.

\section{Submit your next manuscript to BioMed Central and we will help you at every step:}

- We accept pre-submission inquiries

- Our selector tool helps you to find the most relevant journal

- We provide round the clock customer support

- Convenient online submission

- Thorough peer review

- Inclusion in PubMed and all major indexing services

- Maximum visibility for your research

Submit your manuscript at www.biomedcentral.com/submit 